\title{
Review of: "Safety, Immunogenicity and Efficacy of a Recombinant Vesicular Stomatitis Virus Vectored Vaccine Against Severe Fever with Thrombocytopenia Syndrome and Heartland Bandaviruses"
}

\author{
Xianghong Xue $\mathrm{X}^{1}$ \\ 1 Chinese Academy of Agricultural Sciences
}

Potential competing interests: The author(s) declared that no potential competing interests exist.

The authors developed a recombinant VSV (rVSV) expressing the SFTSV glycoproteins Gn/Gc (rVSV-SFTSV) and assessed its safety, immunogenicity and efficacy in mice. The rVSV-SFTSV is safe in immunocompromised mice and is not neuropathogenic when injected intracranially into young immunocompetent mice. Immunization of Ifnar-l- mice with rVSV-SFTSV resulted in high levels of neutralizing antibodies and protection against lethal SFTSV challenge. Passive transfer of sera from immunized $/ \mathrm{fnar}^{-/-}$mice into naïve animals was protective when given pre- or post-exposure. Finally, the immunization with rVSV-SFTSV cross protects mice against challenge with the closely related Heartland virus despite low neutralizing titers to the virus. Thus they indicate that rVSV-SFTSV is a promising vaccine candidate.

Other published paper have evaluated the rVSV-SFTSV, this work declared that they demonstrated the rVSV-SFTSV is not neuropathogenic. However, the neuropathogenicity was only evaluated by clinical signs, which is not precise, and should be furtherly proved by histopathologic examination of brain. I suggest this manuscript would be accepted after supplement the brain histopathologic examination results. 